\title{
Latest Trials Explore \\ Ways to Improve \\ Prostate Cancer \\ Screening
}

Heeral Patel

Editorial Assistant

Citation: EMJ Urol. 2021;9[1]:30-32.

HE EUROPEAN Association of Urology 2021 Congress (EAU21) hosted a session where
six speakers discussed the latest state-of-the-art prostate cancer screening trials. The
experts shared their preliminary results and suggested improvements to the current
screening process.

Prostate cancer (PCa) is the leading cause of cancer-related deaths in males. Patients with PCa can go undiagnosed for many years as the symptoms mostly appear when the tumour has grown large enough to put pressure on the urethra. Currently, prostate-specific antigen (PSA) is used as a marker to help detect and predict PCa onset. However, PSA is not specific to PCa, and PSA is also elevated in other prostatic conditions. This lack of specificity in $\mathrm{PCa}$ screening can lead to over-diagnosis, invasive biopsies, and anxiety in patients.

This highlights the need for improving PCa screening to get an accurate diagnosis and avoid unnecessary procedures. This year's EAU21 Congress invited experts involved in some of the latest state-of-the-art PCa screening trials to discuss their results and the implications for future screening strategies. Six speakers shared their preliminary and ongoing data from clinical trials and their methods for implementing a more efficient PCa screening process.

The first speaker, Monique J. Roobol, Erasmus University Medical Centre, Cancer Institute, Rotterdam, the Netherlands, gave an informative introduction on the burden of PCa screening. Roobol's team developed a PCa screening algorithm that assessed the risk of PSA screening in individuals. The algorithm takes into consideration the geography of the patient and individual risk factors such as age. The algorithm showed that only $10 \%$ of males aged $50-59$ years and only $25 \%$ of males aged $60-70$ years would move on to risk stratification.

\section{The algorithm showed that only 10\% of males aged 50-59 years and only $25 \%$ of males aged 60-70 years would move on to risk stratification.}


The results showed that current methods of PCa testing are not suitable for all individuals, and a tailored approach is urgently needed. Roobol also added that stopping testing in elderly individuals with Iow PSA levels would reduce unnecessary harm and costs. Finally, Roobol shared the vision of the European Cancer Organisation (ECCO) and explained that they aim to provide all males with access to risk-based screening programmes in Europe by 2027.

The next speaker was Christian Arsov, Department of Urology, University Hospital Dusseldorf, Germany, who discussed the preliminary results from the ongoing PROBASE trial. The randomised study took place in four regions of Germany and involved 46,642 males aged 45 years old. There were two study arms: study arm $A$ had an immediate screening of baseline PSA at age 45 years, and study arm B deferred screening until the age of 50 years.

Overall, 344 men were in the high-risk group as they had PSA levels above 3. The participants underwent a second PSA test to confirm the PSA levels, and 179 men were still at risk. The results of the trial showed a very low prevalence of $\mathrm{PCa}$ in 45-year-olds (0.2\%). The speaker emphasised that elevated PSA levels should always be confirmed with a second test.

Over the years, PCa screening has evolved, and the screening steps are as follows: PSA test, systemic biopsy, MRI, and finally, targeted biopsy. At each stage, a percentage of individuals can be ruled out, therefore reducing the chance of overdiagnosis.

Anssi Auvinen from Tampere University, Finland, discussed the ongoing PROSCREEN trial. In this trial, the researchers wanted to add another step to PCa screening called the $4 \mathrm{~K}$ score, a combination of four blood tests, after the PSA test to further eliminate males who are not eligible for an MRI or biopsy. The $4 \mathrm{~K}$ score eliminated $30 \%$ of participants. This trial is still in its infancy and experienced delays due to the pandemic. Calculations show that in 15 years, the researchers should be able to establish the efficacy of adding the $4 \mathrm{~K}$ score to PCa screening.

The next speaker, Tobias Nordstrom, Karolinska Institute, Stockholm, Sweden, also aimed to improve the risk-adapted screening process by combining a genomic test with MRI-targeted

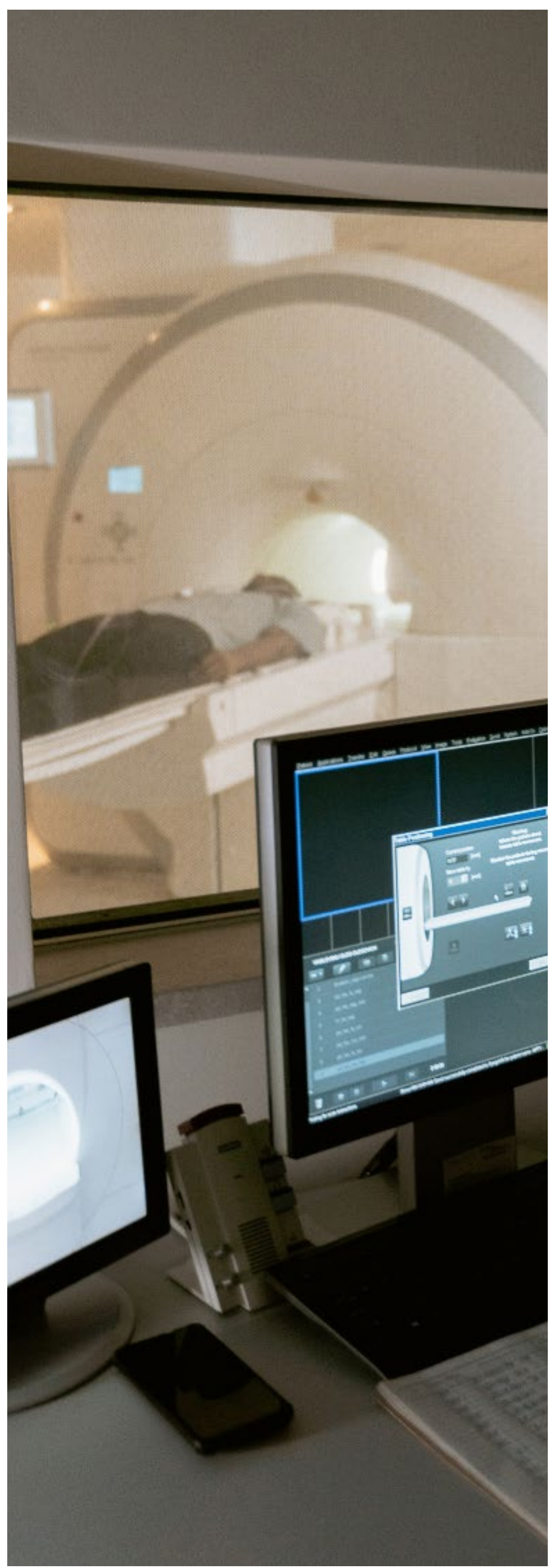


The results showed that

males with normal genetic

risk had a lower chance of

repeated PSA tests, and

males with high genetic

risk were more likely to

have elevated PSA, biopsy,

and a PCa diagnosis.
The primary endpoint was the proportion of males with repeated PSA tests within 2 years. The results showed that males with normal genetic risk had a lower chance of repeated PSA tests, and males with high genetic risk were more likely to have elevated PSA, biopsy, and a PCa diagnosis.

The final speaker, Robert Nam, Sunnybrook Research Institute, Toronto, Canada, discussed the 'Risk-Adapted Screening with MRI Only: MVP Trial.' In PCa screening, MRI is often used alongside PSA tests or genomic tests. However, Nam's group aimed to explore whether MRI could be used as a stand-alone tool to detect PCa. In this Phase III study, patients aged 50 years or older underwent either prostate MRI or PSA test in a randomised 1:1 ratio.

Collectively, 525 were able to participate in this trial: 246 had an MRI, and 248 had a PSA test. Surprisingly, 18 patients dropped out of the study as soon as they found out they would be getting a PSA test as they were upset and were hoping to be in the MRI group. Preliminary results showed that the MRI arm of the study detected more than double the rate of PCa compared to the PSA arm. Nam concluded that MRI is a lot more selective than PSA tests and avoids overtreatment and over-diagnosis. Regardless of initial expenses, it would work out to be more cost-effective in the long term to have an MRI scan rather than repeated PSA tests.

Despite a variety of clinical trials in PCa screening discussed in this year's congress, there was a common theme: a risk-adapted strategy to screening. The speakers proposed novel approaches to get the best out of the screening process whilst reducing harm and over-diagnosis. Many of these trials were in the beginning stages and only showed preliminary results; however, the speakers agreed this was a step in the right direction. In the future, other unanswered questions may be explored such as cost, convenience, and anxiety. Some of the results from these trials will not be confirmed for another decade, but the researchers are optimistic that there will be exciting improvements in $\mathrm{PCa}$ screening in the near future. 